\title{
Association of adult weight gain and nonalcoholic fatty liver in a cross-sectional study in Wan Song Community, China
}

\author{
W.-J. Zhang ${ }^{2}$, L.-L. Chen ${ }^{1}$, J. Zheng ${ }^{1}$, L. Lin ${ }^{1}$, J.-Y. Zhang ${ }^{1}$ and X. Hu ${ }^{1}$ \\ ${ }^{1}$ Department of Endocrinology, Union Hospital, Tongji Medical College, \\ Huazhong University of Science and Technology, Wuhan, China \\ ${ }^{2}$ Department of Hematology, Center Hospital of Wuhan, Tongji Medical College, \\ Huazhong University of Science and Technology, Wuhan, China
}

\begin{abstract}
Our objective was to examine associations of adult weight gain and nonalcoholic fatty liver disease (NAFLD). Cross-sectional interview data from 844 residents in Wan Song Community from October 2009 to April 2010 were analyzed in multivariate logistic regression models to examine odds ratios $(\mathrm{OR})$ and $95 \%$ confidence intervals $(\mathrm{Cl})$ between NAFLD and weight change from age 20. Questionnaires, physical examinations, laboratory examinations, and ultrasonographic examination of the liver were carried out. Maximum rate of weight gain, body mass index, waist circumference, waist-to-hip ratio, systolic blood pressure, diastolic blood pressure, fasting blood glucose, cholesterol, triglycerides, uric acid, and alanine transaminase were higher in the NAFLD group than in the control group. HDL-C in the NAFLD group was lower than in the control group. As weight gain increased (measured as the difference between current weight and weight at age 20 years), the OR of NAFLD increased in multivariate models. NAFLD OR rose with increasing weight gain as follows: OR $(95 \% \mathrm{Cl})$ for NAFLD associated with weight gain of $20+\mathrm{kg}$ compared to stable weight (change $<5 \mathrm{~kg}$ ) was 4.23 (2.49-7.09). Significantly increased NAFLD OR were observed even for weight gains of 5-9.9 kg. For the "age 20 to highest lifetime weight" metric, the OR of NAFLD also increased as weight gain increased. For the "age 20 to highest lifetime weight" metric and the "age 20 to current weight" metric, insulin resistance index (HOMA-IR) increased as weight gain increased $(P<0.001)$. In a stepwise multivariate regression analysis, significant association was observed between adult weight gain and NAFLD $(\mathrm{OR}=1.027,95 \% \mathrm{Cl}=1.002-1.055, \mathrm{P}=0.025)$. We conclude that adult weight gain is strongly associated with NAFLD.
\end{abstract}

Key words: China; Diabetes; Insulin resistance; Lipidosis; Nonalcoholic fatty liver disease; Obesity

\section{Introduction}

Over the past several decades, mainland China has witnessed not only rapid economic growth but also a rapid increase in the incidence of obesity and excess weight, phenomena that tend to accompany such growth. According to the first comprehensive National Health Survey, 60 million (4.6\%) and 200 million (15\%) of the mainland Chinese population were obese and overweight, respectively, in $2004(1,2)$. The increased incidence of obesity and excess weight has led to an increased incidence of obesity-related diseases, including type 2 diabetes and both fatty liver disease and nonalcoholic fatty liver disease (NAFLD). Whereas the incidence of NAFLD in the general population of mainland China almost doubled over the last 10 to 15 years (3), the incidence of NAFLD in the populations of Shanghai and
Wuhan has approximately doubled over only the past 7 to 10 years $(4,5)$. Specifically, it was found to have increased from 13 to $25 \%$ in a sample of residents undergoing routine health examinations in the Shuiguohu district of Wuhan (5) and from 4 to $14 \%$ in a sample of Shanghai BaoSteel Group employees (4).

Emerging as the most common cause of persistently elevated liver enzymes in many countries, NAFLD is frequently associated with metabolic abnormalities related to insulin resistance, including central obesity, dyslipidemia, hypertension, and hyperglycemia. As such, NAFLD is considered a hepatic manifestation of metabolic syndrome. Because the clinical spectrum of NAFLD ranges from bland steatosis with a benign clinical course to cirrhosis with serious complications, including liver

Correspondence: L.-L. Chen, Department of Endocrinology, Union Hospital, Tongji Medical College, Huazhong University of Science and Technology, 1277 Jiefang Ave., Wuhan 430022, Hubei Province, China. Fax: +86-027-8572-6007. E-mail: cllwhxh@aliyun.com 
failure and hepatocellular carcinoma, it is extremely important to identify the risk factors for this disease to assist in its prevention. To contribute to this endeavor, this population-based, cross-sectional study collected interview data from a large sample of residents of the city of Wuhan, China, to examine the relationship between incidence of NAFLD and extent of weight gain after the age of 20 years.

\section{Material and Methods}

\section{Subject selection}

The participants were all residents of Wan Song Community in the city of Wuhan, China, a mature residential community of 8600 men and women aged 40 to 60 years. A multistage-stratified cluster sampling design was used to recruit an approximately equal number of male and female participants and an approximate number of participants aged from 40 to 49 years and from 50 to 60 years between October 2009 and April 2010. A total of 1214 eligible subjects (621 men, 593 women) were able to complete the study. The inclusion criteria for further participation were i) be permanent residents in Wan Song Community and ii) be of ages between 40 and 60 years old. The exclusion criteria were as follows: i) lack of data regarding alcohol consumption, ii) lack of data regarding weight history, iii) loss of more than $5 \mathrm{~kg}$ from the age of 20 years, iv) positive test for hepatitis $B$ virus surface antigen or hepatitis $\mathrm{C}$ virus antibody, v) hepatic enzyme concentration greater than three times the upper normal limit, vi) overt hypo- or hyperthyroidism, vii) cancer, or viii) sonographic evidence of chronic liver disease at baseline. Of the potential subjects, 844 (426 men and 418 women) of a mean age of $50.0 \pm 6.2$ years met all the inclusion criteria and none of the exclusion criteria. All subjects completed a standard questionnaire that collected data on alcohol intake, smoking habits, typical pattern of physical activity, medical history, family history of diabetes, and regular medication use.

The study was approved by the Research Ethics Committee of the Union Hospital, Tongji Medical College, and all participants gave written informed consent.

\section{Subject groups}

In accordance with the experimental methods of Cohen et al. (6), the subjects were divided into four groups according to the extent of weight change since age 20: i) between -5 and $+5 \mathrm{~kg}$, ii) between +5 and $+9.9 \mathrm{~kg}$, iii) between +10 and $+19.9 \mathrm{~kg}$, and iv) $+20 \mathrm{~kg}$ or more. They were also divided into four more groups according to the difference between their maximum weight and weight at age 20 , using the same weight ranges listed above.

\section{Collection of interview data}

The subjects provided written informed consent before participating in a comprehensive, in-person interview collecting various data regarding background, lifestyle, alcohol intake, smoking habits, physical activity, medical history, family history of diabetes, medication use, and weight, including current weight, weight at age 20 years, highest weight (excluding weight during pregnancy), and weight at each life decade $(20,30,40,50$, and 60 years). The maximum rate of weight gain was calculated as the maximum weight change over 10 years divided by the weight before the change.

\section{Collection of clinical and laboratory data}

Height and weight were measured with subjects wearing light clothing without shoes. Body mass index (BMI) was calculated as weight in kilograms divided by the square of the height in meters. Waist circumference (WC) was measured at the midpoint between the lower margin of the last palpable rib and the top of the iliac crest. Hip circumference ( $\mathrm{HC}$ ) was measured around the widest portion of the buttocks. Waist-to-hip ratio (WHR) was calculated as WC divided by HC. Blood pressure was measured with a mercury sphygmomanometer on the right arm with subjects in the sitting position after a 5-min rest.

Blood samples were obtained in the morning after an overnight fast. Fasting plasma glucose (FPG), uric acid (UA), alanine transaminase (ALT), total cholesterol (TC), HDL-C, LDL-C, and triglycerides (TG) were measured. Fasting serum insulin was measured by radioimmunoassay. The insulin resistance index [homeostasis model assessment-insulin resistance (HOMA-IR)] was calculated using the modified HOMA model: (fasting insulin $\times$ fasting glucose)/22.5, which was developed by Haffner et al. (7).

Liver ultrasonography was performed by an experienced ultrasonographist using an Aloka model 1400 realtime ultrasonographic unit with a $3.5-\mathrm{MHz}$ probe (Medical Technologies, Australia).

\section{Diagnosis of NAFLD}

Diagnosis of NAFLD was based on the presence of an ultrasonographic pattern consistent with "bright liver" (brightness and posterior attenuation) with stronger echoes in the hepatic parenchyma than in the renal parenchyma, vessel blurring, and narrowing of the lumen of the hepatic veins in the absence of findings suggestive of other chronic liver disease. Because we had already excluded other known liver diseases, all cases with fatty liver were in accordance with the Chinese diagnostic criteria for NAFLD (alcohol consumption less than $40 \mathrm{~g}$ per week and without consideration of alteration of liver enzymes).

\section{Statistical analysis}

Data are reported as means $\pm S D$. Variables that are not normally distributed, such as the HOMA-IR score, 
were log-transformed before analysis. The unpaired Student $t$-test for continuous variables or the chi-square test for percentages was used to compare groups. Multivariate logistic regression analysis was performed to determine the association of adult weight gain with NAFLD. All statistical analyses were performed using the SPSS 13.0 software package (USA). Results with $P$ values less than 0.05 were considered to be statistically significant.

\section{Results}

\section{Characteristics of study population}

Maximum rate of weight gain, BMI, WHR, systolic blood pressure (SBP), diastolic blood pressure (DBP), FPG, TC, TG, UA, and ALT in the NAFLD group were higher than in the control group $(P<0.01)$, and HDL-C in the NAFLD group was lower than in the control group (Table 1).

Multivariate-adjusted odds ratios $(95 \% \mathrm{Cl})$ were used for the NAFLD group by category of adult weight change among 844 residents.

Table 1. Clinical characteristics of the study subjects.

\begin{tabular}{lcc}
\hline & NAFLD group & Control group \\
\hline $\mathrm{N}$ & 215 & 629 \\
Age (years) & $50.36 \pm 6.5$ & $49.9 \pm 6.1$ \\
Gender (male) & 128 & 305 \\
BMI (kg/m²) & $27.4 \pm 3.6$ & $23.9 \pm 3.0$ \\
WC (cm) & & \\
Male & $93.1 \pm 7.9^{*}$ & $84.7 \pm 7.3$ \\
Female & $89.5 \pm 6.2^{*}$ & $81.1 \pm 5.9$ \\
WHR & & \\
Male & $0.93 \pm 0.06^{*}$ & $0.88 \pm 0.05$ \\
Female & $0.89 \pm 0.05^{*}$ & $0.86 \pm 0.03$ \\
SBP (mmHg) & $129.7 \pm 19.1^{*}$ & $120.6 \pm 19.0$ \\
DBP (mmHg) & $85.0 \pm 11.7^{*}$ & $79.1 \pm 11.3$ \\
FPG (mM) & $5.43 \pm 1.22^{*}$ & $4.94 \pm 0.96$ \\
TC (mM) & $5.11 \pm 0.99^{*}$ & $4.88 \pm 0.98$ \\
TG (mM) & $2.14 \pm 1.17^{*}$ & $1.64 \pm 1.27$ \\
HDL-C (mM) & $1.31 \pm 0.29^{*}$ & $1.58 \pm 0.42$ \\
LDL-C (mM) & $2.87 \pm 0.84$ & $2.77 \pm 0.74$ \\
UA ( $\mu$ M) & $328.6 \pm 84.5^{*}$ & $301.6 \pm 90.6$ \\
ALT (U/L) & $37.9 \pm 21.6^{*}$ & $27.7 \pm 20.2$ \\
Maximum rate of & $17.40 \pm 15.08^{*}$ & $9.51 \pm 12.01$ \\
weight gain $(\%)$ & & \\
\hline
\end{tabular}

Data are reported as means \pm SE. NAFLD: nonalcoholic fatty liver disease; BMI: body mass index; WC: waist circumference; WHR: waist-to-hip ratio; SBP: systolic blood pressure; DBP: diastolic blood pressure; FPG: fasting plasma glucose; TC: total cholesterol; TG: triglycerides; HDL-C: high-density lipoprotein cholesterol; LDL-C: low-density lipoprotein cholesterol; UA: uric acid; ALT: alanine transaminase. ${ }^{*} \mathrm{P}<0.01$ vs control group (unpaired Student $t$-test and chi-square test).
The proportion of participants who were overweight or obese $\left(\mathrm{BMI}>25 \mathrm{~kg} / \mathrm{m}^{2}\right)$ at enrollment was $49.8 \%$. At age 20 , a much higher proportion $(97 \%)$ of the participants were healthy or thin $\left(\mathrm{BMl}<25 \mathrm{~kg} / \mathrm{m}^{2}\right)$. Most $(84.3 \%)$ of the participants reported gaining weight since age 20 . As weight gain increased (measured as the difference between current weight and weight at age 20), the odds ratios (OR) of NAFLD increased in multivariate models. NAFLD OR rose with increasing weight gain: OR $(95 \% \mathrm{Cl})$ for NAFLD associated with weight gain of $25+\mathrm{kg}$ compared to stable weight (change $<5 \mathrm{~kg}$ ) was 4.23 (2.49-7.09). Significantly increased NAFLD OR were observed even for weight gains of $5-9.9 \mathrm{~kg}$. For the "age 20 to highest lifetime weight" metric, the OR of NAFLD also increased as weight gain increased (Table 2).

\section{HOMA-IR by category of adult weight change among 844 residents}

For the age 20 to highest lifetime weight and the "age 20 to current weight" metric, HOMA-IR is the significant difference between each group and increased as weight gain increased $(\mathrm{P}<0.001$; Figure 1).

\section{Stepwise multivariate regression analysis of NAFLD}

Stepwise multivariate regression analysis adjusted for rate of maximum weight change (calculated as maximum weight change over 10 years divided by weight before change), a history of hyperglycemia and hyperlipidemia, BMI, WHR, TC level, TG level, FPG level, and HOMA-IR score revealed that $\mathrm{BMI}(\beta=1.252, \mathrm{P}<0.001), \mathrm{FPG}$ level $(\beta=1.623, P<0.05)$, HOMA-IR score $(\beta=3.001$, $P<0.01)$, and TG level $(\beta=1.18, P<0.05)$ were significantly associated with rate of maximum weight change. The incidence of NAFLD was also found to be significantly associated with rate of maximum weight change $(\beta=1.027, \mathrm{P}<0.05$; Table 3$)$.

\section{Discussion}

In this cross-sectional study of 844 residents of a mature residential community in the city of Wuhan, a decrease in HDL-C levels and varying degrees of increases in BMI, WC, WHR, SBP, DBP, TC level, TG level, UA level, and ALT level were observed in subjects with NAFLD compared to subjects without NAFLD, a finding that is in accordance with previous studies. A more significant finding is that adult weight gain is strongly associated with the incidence of NAFLD, as evidenced by the observation that i) the rate of maximum weight gain was significantly greater in subjects with NAFLD compared to subjects without NAFLD; ii) the OR of having NAFLD increased with an increasing difference between current weight and weight at age 20 after multifactor adjustments; iii) the OR of having NAFLD increased with an increasing difference between maximum weight and weight at age 20 after multifactor adjustments, and iv) a 
Table 2. Multivariate-adjusted odds ratios $(95 \% \mathrm{Cl})$ for nonalcoholic fatty liver disease by category of adult weight change among 844 residents in Wansong Community from October 2009 to April 2010.

\begin{tabular}{lcccc}
\hline & $\mathrm{N}$ & $\mathrm{OR}$ & $95 \% \mathrm{Cl}$ & $\mathrm{P}$ \\
\hline Weight gain [current-age 20] (kg) & & & & \\
$\quad$ Stable & 183 & 1.0 & Ref. & $<0.001$ \\
$\quad$ Gained 5-14.9 kg & 328 & 1.26 & $(1.03-2.54)$ & $<0.001$ \\
$\quad$ Gained 15-24.9 kg & 227 & 2.45 & $(2.49-7.09)$ & $<0.001$ \\
$\quad$ Gained 25+ kg & 106 & 4.23 & & \\
Weight gain [most-age 20] (kg) & 171 & 1.0 & $(1.26-3.54)$ & $<0.001$ \\
$\quad$ Stable & 327 & 1.94 & $(2.17-6.06)$ & $<0.001$ \\
$\quad$ Gained 5-14.9 kg & 243 & 3.60 & $(4.49-9.09)$ & $<0.001$ \\
$\quad$ Gained 15-24.9 kg & 103 & 5.23 & & \\
$\quad$ Gained 25+ kg & & & \\
\hline
\end{tabular}

Adjusted for age, gender, BMl at age 20. Stable weight change is gain or loss of less than $5 \mathrm{~kg}$. Multivariate logistic regression analysis was used for statistical analyses.

statistically significant relationship was found between rate of maximum weight gain $(\mathrm{OR}=1.016,95 \% \mathrm{Cl}=$ 1.002-1.031) and incidence of NAFLD corrected by BMI, WHR, history of hyperlipidemia and diabetes, FPG level, HOMA-IR score, TC level, and TG level, all of which indicate that adult weight gain is associated with incidence of NAFLD.

Although many studies have attempted to elucidate the relationship between obesity and NAFLD, few have examined the relationship between adult weight gain and NAFLD. Among the few that have, a 5-year prospective study of 2895 Koreans aged between 20 and 79 years found that the weight change is closely related to the course of NAFLD (8). Although the study concluded that weight gain can increase the severity of NAFLD, it did not rule out the effect of current weight on incidence of NAFLD. In a retrospective analysis of physical examination data collected from 5860 Chinese workers, Fan et al.

(9) found that the presence of obesity and related metabolic disorders at baseline and the increase in weight and fasting serum TG levels at follow-up are important predictors in the development of NAFLD. In contrast, the
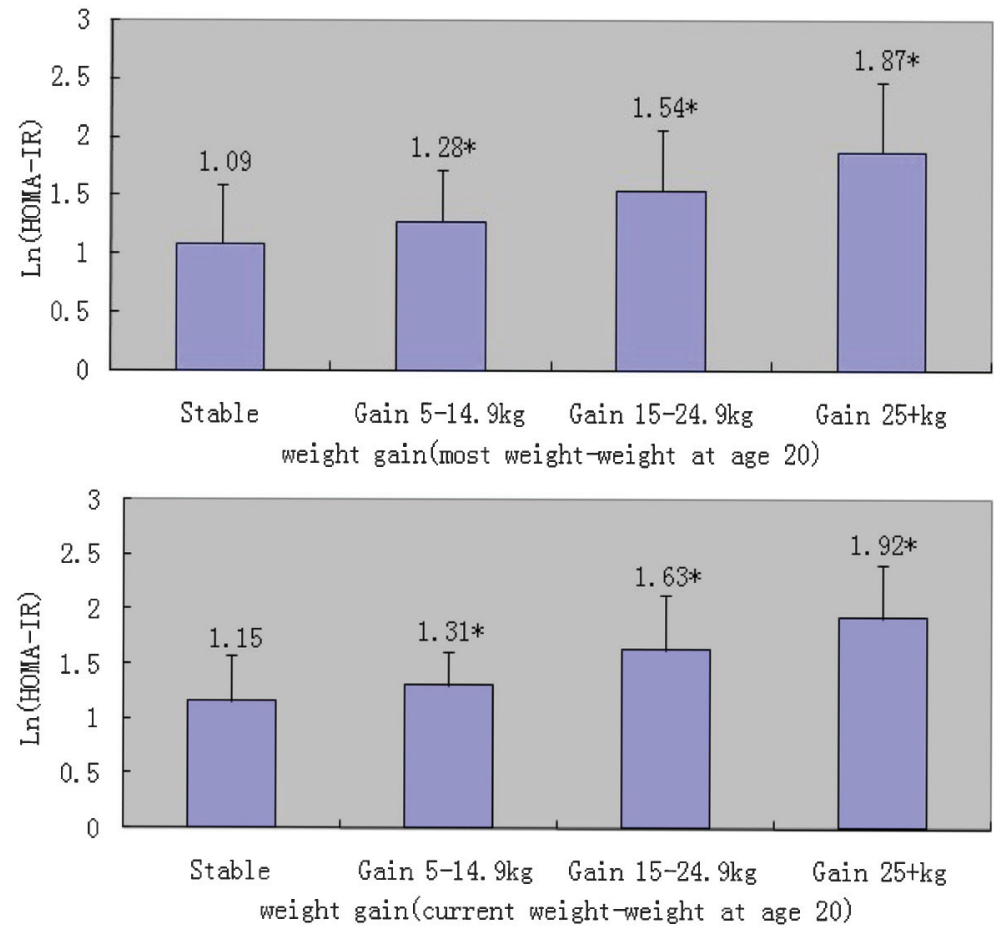

Figure 1. HOMA-IR by category of adult weight change among 844 residents in Wansong Community from October 2009 to April 2010. Adjusted for age, gender, and BMl at age 20. Stable weight change is gain or loss of less than $5 \mathrm{~kg}$. HOMA-IR was log-transformed before analysis for it was not normally distributed. ${ }^{*} \mathrm{P}<0.05$ vs stable group (unpaired Student $t$ test). 
Table 3. Independently associated factors of nonalcoholic fatty liver disease.

\begin{tabular}{lccc}
\hline Variables & $\mathrm{P}$ & Adjusted OR & $95 \% \mathrm{Cl}$ \\
\hline BMI & $<0.001$ & 1.252 & $1.105-1.418$ \\
FPG & 0.02 & 1.623 & $1.081-2.438$ \\
HOMA-IR & 0.004 & 3.001 & $1.417-6.365$ \\
TG & 0.018 & 1.182 & $1.029-1.357$ \\
Maximum weight growth rate & 0.025 & 1.027 & $1.002-1.055$ \\
\hline
\end{tabular}

Adjusted for history of hyperlipidemia and hyperglycemia, body mass index (BMI), waist-to-hip ratio (WHR), cholesterol, triglyceride (TG), insulin resistance index (HOMA-IR), fasting plasma glucose (FPG). Multivariate logistic regression analysis was used for statistical analyses.

current study found that adult weight gain is associated with NAFLD, which is independent of current weight.

Several studies have reported that adult weight gain is an independent risk factor for insulin resistance-related diseases, such as type 2 diabetes, coronary atherosclerotic heart disease, and hypertension. Among them, a prospective study following 6789 men for 20 years found that adult weight gain is associated with increased risk of cardiovascular disease and diabetes, whereas weight loss is associated with significant reduction in risk of cardiovascular disease and diabetes, regardless of whether individuals are overweight or normal weight at baseline (10). Likewise, a cross-sectional study found a strong positive association between weight gain and OR of developing diabetes, such that the relative risk for diabetes increased with an increase in adult weight gain (6). A prospective study following 82,473 American women for 16 years found that adult weight gain remains closely related to the development of hypertension, even after the effect of current BMI has been excluded (11). These findings indicate that insulin resistance is significantly involved in the pathogenic mechanism underlying NAFLD, playing a central role as an initiator of the "first hit" according to the widely accepted "two-hit hypothesis" (12). Based on the observation that the HOMA-IR score increased with an increase in body weight after multifactor adjustments in the current study, we hypothesized that insulin resistance might play an important role in the development of NAFLD induced by adult weight gain.

The role of adult weight gain in the pathogenic mechanism underlying the development of NAFLD remains unclear. Rapid improvement in nutrition can induce substantial increases in weight within a short period, which is often followed by excessive growth of body fat. One animal study reported a rapid increase in the weight of rats that resumed normal feeding after a period of dietary restriction (13). Moreover, although the

\section{References}

1. Wu Y. Overweight and obesity in China. The once lean giant has a weight problem that is increasing rapidly. BMJ 2006; final weight of these rats was lower than that of rats in a control group, they were found to have more obvious hepatic lipidoses and decreased insulin sensitivity compared to the control group. This finding indicates that the development of hepatic lipidoses is strongly associated not with weight gain itself but rather with rapid weight gain. The mechanism underlying fat deposition in rat viscera induced by rapid improvement of nutrition after malnutrition may be related to increased secretion of gastrin and increased expression of the gastrin receptor in fat cells, the latter of which may be involved in the development of insulin resistance (14).

The observation of an increase in the incidence of NAFLD with an increase in the rate of weight gain in the subjects examined in this study strongly indicates that adult weight gain is an independent risk factor for the development of NAFLD. Comparison of the study findings with those of past studies leads us to hypothesize that the rapid increase in the incidence of NAFLD in China might be related to the rapid improvement of nutrition. Further research is now necessary to test this hypothesis.

This study faced several limitations that may affect the reliability and generalizability of the results. First, the data regarding weight history were self-reported by the subjects, who, especially those who were overweight or obese, may have underestimated the extent of their weight gain over the years. However, evidence is accumulating that recall of adolescent weight by subjects is a reliable means of data collection when it is impossible to directly measure past weight in an epidemiological investigation (15). Second, the sample was composed of the residents of only one residential center, which may limit application of the findings to other populations. To address these limitations and further explore the relationship between weight change and NAFLD, we hope to conduct a prospective multiplecenter study of these factors. 
et al. Prevalence of the metabolic syndrome and overweight among adults in China. Lancet 2005; 365: 1398-1405, doi: 10.1016/S0140-6736(05)66375-1.

3. Fan JG, Farrell GC. Epidemiology of non-alcoholic fatty liver disease in China. J Hepatol 2009; 50: 204-210, doi: 10.1016/j.jhep.2008.10.010

4. Fan JG, Li F, Cai XB, Peng YD, Ao QH, Gao Y. The importance of metabolic factors for the increasing prevalence of fatty liver in Shanghai factory workers. J Gastroenterol Hepatol 2007; 22: 663-668, doi: 10.1111/j.1440-1746.2007.04892.x.

5. Wang Z, Xia B, Ma C, Hu Z, Chen X, Cao P. Prevalence and risk factors of fatty liver disease in the Shuiguohu district of Wuhan city, central China. Postgrad Med J 2007; 83: 192195, doi: 10.1136/pgmj.2006.052258.

6. Cohen SS, Signorello LB, Blot WJ. Adult weight gain and diabetes among African American and white adults in southeastern US communities. Prev Med 2009; 49: 476481, doi: 10.1016/j.ypmed.2009.10.010.

7. Haffner SM, Kennedy E, Gonzalez C, Stern MP, Miettinen $\mathrm{H}$. A prospective analysis of the HOMA model. The Mexico City Diabetes Study. Diabetes Care 1996; 19: 1138-1141, doi: $10.2337 /$ diacare.19.10.1138.

8. Kim HK, Park JY, Lee KU, Lee GE, Jeon SH, Kim JH, et al. Effect of body weight and lifestyle changes on long-term course of nonalcoholic fatty liver disease in Koreans. Am J Med Sci 2009; 337: 98-102, doi: 10.1097/MAJ.0b013e3181812879.

9. Fan JG, Zhou Q, Wo QH. [Effect of body weight mass and its change on the incidence of nonalcoholic fatty liver disease]. Zhonghua Gan Zang Bing Za Zhi 2010; 18: 676679.

10. Wannamethee SG, Shaper AG, Walker M. Overweight and obesity and weight change in middle aged men: impact on cardiovascular disease and diabetes. J Epidemiol Community Health 2005; 59: 134-139, doi: 10.1136/jech.2003.015651.

11. Huang Z, Willett WC, Manson JE, Rosner B, Stampfer MJ, Speizer FE, et al. Body weight, weight change, and risk for hypertension in women. Ann Intern Med 1998; 128: 81-88, doi: 10.7326/0003-4819-128-2-199801150-00001.

12. Marchesini G, Brizi M, Bianchi G, Tomassetti S, Bugianesi $\mathrm{E}$, Lenzi M, et al. Nonalcoholic fatty liver disease: a feature of the metabolic syndrome. Diabetes 2001; 50: 1844-1850, doi: $10.2337 /$ diabetes.50.8.1844.

13. Chen LL, Hu X, Zheng J, Kong W, Zhang HH, Yang WH, et al. Lipid overaccumulation and drastic insulin resistance in adult catch-up growth rats induced by nutrition promotion after undernutrition. Metabolism 2011; 60: 569-578, doi: 10.1016/j.metabol.2010.05.014.

14. HQ Li, Chen LL, Deng XL, Zhang JY, Liao YF, Liu ZH, et al. Changes of serum gastrin and CCK2R expression of visceral adipocytes in catch-up rats. Chin $J$ Endocrinol Metab 2011; 27: 607-609.

15. Stevens J, Keil JE, Waid LR, Gazes PC. Accuracy of current, 4-year, and 28-year self-reported body weight in an elderly population. Am J Epidemiol 1990; 132: 1156-1163. 